\title{
On the Role of Compensatory Operators in Fuzzy Result Merging for Metasearch
}

\author{
Arijit De \\ Tata Consultancy Services, Mumbai, India \\ arijit.axd9142@gmail.com
}

\begin{abstract}
A key metasearch engine task is result merging of search results from multiple search engines in response to a user query. The problem of result merging has been widely studied as a multi-criteria decision making model (MCDM). While many MCDM techniques have been employed to create experimental models for result merging, the most notable have used fuzzy aggregation operators such as the OWA operators and its extensions and variations. In this work we study the role of applying fuzzy algebraic t-norms, s-norms and compensatory operators in fuzzy result merging for metasearch. Our results will demonstrate the superiority of compensatory operators over tnorm aggregation functions in the context of result merging for metasearch.
\end{abstract}

Keywords: Information Retrieval, Metasearch Engines, Fuzzy Sets, Fuzzy Aggregation Operators.

\section{Introduction}

A metasearch engine is a search engine that can be used to search multiple search engines systems concurrently. Metasearch engines are particularly useful in searching through topic specific search systems like PUBMED, MEDILE etc. A typically search engine output comprises of a list of results (documents/URLs/database records), ranked in the order of relevance. However, different search engines evaluate and consequently rank results differently. The problem of result merging is to aggregate the rankings of each result to come up with a composite rank, such that the final ranking preserves the order of relevance. In this paper we propose two models Compensatory Ordered Weights Average (COWA) and Importance Guided Compensatory Ordered Weights Average (IGOWA) models for result aggregation using the OWA operator [11] and compensatory aggregation function [15]. We compare our models with the existing OWA [5], IGOWA, t-norm OWA and t-norm IGOWA [3, 4]. This paper is organized as follows. In Section 2, we discuss previous models for result merging, including a discussion on the OWA, IGOWA, t-norm OWA and t-norm IGOWA models. In Section 3 we describe our proposed models, COWA and IGCOWA. In Section 4 we describe experiments comparing COWA and IGCOWA with OWA, IGOWA and t-norm IGOWA and discuss our results. In Section 5 summarize our findings in a conclusion. 


\section{Previous Work}

In early work on aggregation, includes the work of Fox and Shaw [6, 7] and Aslam and Montague [1]. The latter proposed two models Borda-Fuse and Weighted BordaFuse based on the political election strategy, Borda Count [2].

Diaz [5] developed the first fuzzy result aggregation model OWA, based on Yager Ordered Weighted Average (OWA) [11, 12] operator. The OWA model uses a positional value (PV) to quantify the rank of a result in a result list. The positional value (PV) of a result ranked $r$ in a result list is $(n-r+1)$ where $n$ is the total number of results in the list. The OWA model uses the OWA operator to aggregate the PVs of each result. Let us say we have $n$ criteria and an alternative $x$. Let $a_{i}$ represent the degree to which $x$ satisfies the $i^{\text {th }}$ criteria. Thus we have a set $\left\{a_{1} \ldots a_{n}\right\}$. Let $b_{j}$ is the $j^{\text {th }}$ largest value within the set $\left\{a_{1}, a_{2}, \ldots, a_{n}\right\}$. Then $F$ (eq. 1) defines the OWA operator.

$$
F\left(a_{1}, a_{2}, a_{3}, \ldots, a_{n}\right)=\sum_{j=1}^{n} w_{j} b_{j}
$$

In the OWA model for metasearch, the PV (or inverse of rank) is considered extent to which the result (alternative) satisfies a search engine (criteria). The ordered weights are computed using a linguistic RIM quantifier $Q(r)=r^{\alpha}$ as described in equation (2). The orness associated with the quantifier, orness $(Q)=(1+\alpha)^{-1}$. Experiments in $[10,11]$ demonstrate the OWA model outperforms the Borda Fuse and Weighted Borda Fuse models.

$$
\mathrm{w}_{\mathrm{i}}=\mathrm{Q}\left(\frac{\mathrm{i}}{\mathrm{n}}\right)-\mathrm{Q}\left(\frac{\mathrm{i}-1}{\mathrm{n}}\right)
$$

The OWA model however, does not consider search engine importance weights in result aggregation. To overcome this, De [3, 4] proposed the IGOWA (importance guided) model for metasearch. The IGOWA model uses Yager's [12] Importance Guided Aggregation technique to generate the ordered weights for aggregation using the OWA operator. Once again PVs of results from result lists are aggregated using the OWA operator. However, weights are generated as per equation (3) below. Let the importance weight for the $\mathrm{i}^{\text {th }}$ criteria be $\mathrm{V}_{\mathrm{i}}$. Let $\mathrm{V}_{\mathrm{i}} \varepsilon[0,1]$. For an alternative $\mathrm{x}$ there will be a pair $\left(\mathrm{V}_{\mathrm{i}}, \mathrm{a}_{\mathrm{i}}\right)$ for each criteria $\mathrm{i}$. The criteria scores can be sorted in descending order with $b_{k}$ being the $k^{\text {th }}$ largest $a_{i}$. Let $u_{k}$ be the importance weight attached to $b_{k}$. We can now associate, with alternative $x$, a collection of $n\left(u_{k}, b_{k}\right)$ pairs, where the $b_{k}$ 's are degrees to which $x$ satisfies the $n$ criteria in descending order. The ordered weights can now be obtained using equation (3) and where $\mathrm{T}=\sum_{\mathrm{j}=1}^{\mathrm{n}} \mathrm{u}_{\mathrm{j}}$. Yager [12] proposes a set of extensions of the OWA operator, which he calls the triangular norm (t-norm) OWA operators. This is essentially a quantifier guided aggregation decision function that strikes a balances the RIM quantifier Q, defined previously, that stipulates the degree of satisfaction that is attained when satisfying $\mathrm{i}^{\text {th }}$ criteria with the need to find $i$ criteria that are satisfied. Combining these two factors, Yager arrives at the aggregation function shown in eq. (4). Here $b_{j}$ is $j^{\text {th }}$ greatest $a_{i}$ and $T$ is a t-norm function. 


$$
\begin{aligned}
& w_{k}(x)=Q\left(\frac{\sum_{j=1}^{k} u_{j}}{T}\right)-Q\left(\frac{\sum_{j=1}^{k-1} u_{j}}{T}\right) \\
& F\left(a_{1}, a_{2}, a_{3}, \ldots, a_{n}\right)=\sum_{j=1}^{n} w_{j} T\left(b_{1}, . ., b_{j}\right)
\end{aligned}
$$

De [3, 4] used an algebraic t-norm eq. (5) to propose two models for result merging. The first was the t-norm OWA result merging model for metasearch and did not consider search engine importance weights. The second was the importance guided t-norm IGOWA model that use search engine weights to generate ordered weights using equation (3). In each case multiple PVs for each result (obtained from different search engine result lists) are obtained. Experiments of De [3, 4] demonstrated that when using an algebraic t-norm the t-norm OWA model outperformed the OWA model and the t-norm IGOWA model outperformed the IGOWA model for metasearch. The IGOWA and t-norm OWA model both outperformed the OWA model in creating improved relevance order ranking.

$$
\mathrm{T}\left(\mathrm{b}_{1}, \ldots \ldots \mathrm{b}_{\mathrm{n}}\right)=\prod_{\mathrm{i}=1}^{\mathrm{n}} \mathrm{b}_{i}
$$

\section{Proposed Models}

Several researchers working in the area of fuzzy decision making noticed that t-norms and their dual s-norms lack the compensation behavior crucial to any aggregation process. Zimmermann and Zysno [15] were the first to notice that in a decision function making context humans neither follow the behavior of a t-norm or an s-norm strictly when aggregating. Zimmermann and Zysno [15] proposed an aggregation function on the unit interval based on t-norms and s-norms as described in equation (6). Here $\gamma$ is the extent of compensation provided. Yager [14] proposes a function to calculate the value of $\gamma$ as described in equation (7).

The motivation of our work was to study how using compensatory operators, affects the result of merging result merging for metasearch. We build two models. Our first model was the Compensatory Ordered Weighed Aggregation (COWA) model. This model employs the Zimmermann [15] compensatory aggregation function in result aggregation using the OWA operator as defined in eq. (8). This model does not take into consideration search engine weights in result merging. In our model we use the algebraic t-norm for aggregation as described in eq. (5). We compare the performance of this model to the OWA model [5] and t-norm OWA model $[3,4]$.

Our second model is the Importance Guided Compensatory Ordered Weighted Aggregation (IGCOWA) model for result merging. The model is similar as it user the 
Zimmerman [15] aggregation function in conjunction with the OWA operator as described in eq. (8). However the ordered weights for aggregation are generated using equation (3) as in IGOWA and t-norm IGOWA [3, 4]. Both our models use a Regular Increasing Monotone (RIM) quantifier as described earlier of the form $Q(r)=r^{\alpha}$ as a function to generate ordered weights.

$$
\begin{gathered}
\mathrm{Z}_{\gamma}\left(\mathrm{b}_{1}, \mathrm{~b}_{2}, \mathrm{~b}_{3}, \ldots, \mathrm{b}_{\mathrm{n}}\right)=\left(\prod_{\mathrm{i}=1}^{\mathrm{n}} \mathrm{b}_{\mathrm{i}}\right)^{1-\gamma} \bullet\left(1-\prod_{\mathrm{i}=1}^{\mathrm{n}}\left(1-\mathrm{b}_{\mathrm{i}}\right)\right)^{\gamma} \\
\gamma=\frac{\mathrm{T}\left(\mathrm{b}_{1}, \ldots, \mathrm{b}_{\mathrm{n}}\right)}{\mathrm{T}\left(\mathrm{b}_{1}, \ldots, \mathrm{b}_{\mathrm{n}}\right)+\mathrm{T}\left(1-\mathrm{b}_{1}, \ldots, 1-\mathrm{b}_{\mathrm{n}}\right)} \\
\mathrm{F}\left(\mathrm{a}_{1}, \mathrm{a}_{2}, \mathrm{a}_{3}, \ldots, \mathrm{a}_{\mathrm{n}}\right)=\sum_{\mathrm{j}=1}^{\mathrm{n}} \mathrm{w}_{\mathrm{j}} \mathrm{Z}_{\gamma}\left(\mathrm{b}_{1}, . ., \mathrm{b}_{\mathrm{j}}\right)
\end{gathered}
$$

\section{$4 \quad$ Experiments and Results}

For our experiments, we use Hersh's [8] OHSUMED collection within the LETOR 2 (Learning TO Rank) [9] dataset from Microsoft Research Asia. The LETOR2 dataset comprises of a collection of OHSUMED documents (results), a query set of size of 106, a set of 25 algorithms (search engines) that are used to judge the relevance of each document to each query. For our experiments, our performance metric is RecallBased (RB) Precision as defined by Bollmann and Raghavan [16]. We compare our proposed models COWA and IGCOWA against existing models OWA, t-norm OWA, IGOWA and t-norm IGOWA. Odd numbered queries are used for learning search engine importance weights based on performance of search engines over the query set. For our experiments for each odd numbered query, we randomly pick $\mathrm{N}$ search engines from the 25 available. The value of $\mathrm{N}$ is varied from 2 to 12 . Overall 1000 sets of experiments are done for each value of $\mathrm{N}$. Table 1 shows the results. We compute the average precision at recall levels of $0.25,0.5,0.75$ and 1 . The results are shown in Table 1 and Table 2.

Table 1. Results comparing COWA, IGCOWA vs. OWA, IGOWA, t-norm OWA and t-norm IGOWA when number of lists varies from 2 to 12 .

\begin{tabular}{ccccccc}
\hline $\begin{array}{c}\mathrm{N} \\
\text { (Number } \\
\text { of lists } \\
\text { merged) }\end{array}$ & OWA & IGOWA & $\begin{array}{c}\text { t-norm } \\
\text { OWA }\end{array}$ & $\begin{array}{c}\text { t-norm } \\
\text { IGOWA }\end{array}$ & COWA & IGCOWA \\
\hline 2 & 0.4051 & 0.4231 & 0.4233 & 0.4472 & 0.4538 & 0.4638 \\
4 & 0.4237 & 0.4445 & 0.4453 & 0.4491 & 0.4573 & 0.4783 \\
6 & 0.4297 & 0.4593 & 0.4597 & 0.4638 & 0.4791 & 0.4891 \\
8 & 0.4332 & 0.4682 & 0.4712 & 0.4783 & 0.5011 & 0.5013 \\
10 & 0.4681 & 0.4783 & 0.4813 & 0.4891 & 0.5113 & 0.5291 \\
12 & 0.4732 & 0.4813 & 0.4913 & 0.5013 & 0.5231 & 0.5345 \\
\hline
\end{tabular}


Table 2. Results comparing COWA, IGCOWA vs. OWA, IGOWA, t-norm OWA and t-norm IGOWA when orness of aggregation varies from 0.8 to 0.2 .

\begin{tabular}{ccccccc}
\hline $\begin{array}{c}\text { Orness } \\
(\mathrm{O})\end{array}$ & OWA & IGOWA & $\begin{array}{c}\text { Average Precision of the Merged List } \\
\text { t-nowA }\end{array}$ & $\begin{array}{c}\text { t-norm } \\
\text { IGOWA }\end{array}$ & COWA & IGCOWA \\
& & & & & \\
\hline $\mathrm{O} \geq 0.8$ & 0.4371 & 0.4413 & 0.4417 & 0.4472 & 0.5146 & 0.5292 \\
$0.8 \geq \mathrm{O} \geq 0.6$ & 0.4251 & 0.4345 & 0.4453 & 0.4491 & 0.4629 & 0.5177 \\
$0.6 \geq \mathrm{O} \geq 0.4$ & 0.4108 & 0.4139 & 0.4397 & 0.4428 & 0.4439 & 0.5237 \\
$0.4 \geq \mathrm{O} \geq 0.2$ & 0.4332 & 0.4428 & 0.4712 & 0.4783 & 0.5211 & 0.5378 \\
\hline
\end{tabular}

\section{Conclusions}

From Table 1 it is clear that the COWA model outperforms the models in its class (without search engine importance weights), namely the OWA and the t-norm OWA (with algebraic t-norms) models. The overall improvements over t-norm OWA model are $7.20,2.69,4.22,6.34,6.23$ and $6.47 \%$ when merging 2, 4 6, 8, 10 and 12 search engines respectively. Similarly, in its class of models (requiring search engine importance weights) IGCOWA model improves upon the IGOWA model by 3.7, 6.5, $5.45,4.80,8.17$ and $6.62 \%$ when merging 2, 4, 6, 8, 10 and 12 search engines. . In Table 1 it is also observed that as the number of search engines increases the overall performance in terms of average precision increases. This is primarily because when more search engine results are merged more relevant results are present in the merged list.

Orness is a key measure in fuzzy aggregation. For the OWA, t-norm OWA and COWA models orness is computed as $1 /(1+\alpha)$. Here $\alpha$ is the parameter of the RIM quantifier $\mathrm{Q}(\mathrm{r})=\mathrm{r}^{\alpha}$ used to compute ordered weights. We also measured the performance of aggregation for different levels of orness. In previous findings $[3,4,5]$ it was observed that as orness of aggregation decreases, the performance in terms of average precision decreases, till averaging conditions are achieved. When orness $=0.5$ then the average precision falls to its lowest value. Following this as orness decreases and andness increases the average precision increases.

This is consistent with findings in [3, 4 and 5] illustrated in results from Table 2. Also for each level of orness the performance of COWA is better than that of algebraic t-norm OWA and OWA. For the models that require importance weights the IGCOWA model also outperforms the t-norm IGOWA and IGOWA models.

The overall improvements of COWA over OWA and t-norm OWA are $9.98 \%$ and $8.01 \%$. The overall improvements of IGCOWA over IGOWA and t-norm IGOWA are $5.52 \%$ and $5.87 \%$. Clearly compensatory operators clearly improve the performance of aggregation in terms of average precision. 


\section{References}

1. Aslam, J., Montague, M.: Models for metasearch. In: Proceedings of the 24th Annual International ACM SIGIR Conference on Research and Development in Information Retrieval (SIGIR 2001), New Orleans, LA, USA, September 1-6, pp. 276-284. ACM Press, New York (2001)

2. Borda, J.C.: Memoire sur les elections au scrutiny. Histoire de l'Academie Royale des Sciences, Paris (1781)

3. De, A., Diaz, E.D., Raghavan, V.V.: On Fuzzy Result Merging for Metasearch. In: IEEE International Conference on Fuzzy Systems, London, United Kingdom. IEEE Press (July 2007)

4. De, A.: Fuzzy result merging models for metasearch, A Dissertation. University of Louisiana Press (2006)

5. Diaz, E.D., De, A., Raghavan, V.V.: A comprehensive OWA-based framework for result merging in metasearch. In: Ślęzak, D., Yao, J., Peters, J.F., Ziarko, W.P., Hu, X. (eds.) RSFDGrC 2005. LNCS (LNAI), vol. 3642, pp. 193-201. Springer, Heidelberg (2005)

6. Fox, E.A., Shaw, J.A.: Combination of multiple searches: Part 2. In: Proceedings of the 2nd Text Retrieval Conference (TREC-2), Gaithersburg, MD, August 31-September 2, pp. 243-252. US Government Printing Office, Washington DC (1993)

7. Fox, E.A., Koushik, M.P., Shaw, J.A., Modlin, R., Rao, D.: Combination of multiple searches: Part 1. In: Proceedings of the 1st Text Retrieval Conference (TREC-1), Gaithersburg, MD, USA, November 4-6, pp. 319-328. US Government Printing Office, Washington DC (1992)

8. Hersh, W., Buckley, C., Leone, T.J., Hickam, D.: OHSUMED: An interactive retrieval evaluation and new large test collection for research. In: Proceedings of the 17th Annual International ACM SIGIR Conference on Research and Development in Information Retrieval, pp. 192-201. ACM/Springer, New York, NY (1994)

9. Liu, T., Xu, J., Qin, T., Xiong, W., Li, H.: LETOR 2007: Benchmark dataset for re-search on learning to rank for information retrieval. In: LR4IR 2007, in Conjunction with SIGIR 2007 (2007)

10. Turksen, I.B.: Interval-valued fuzzy sets and 'compensatory AND'. Fuzzy Sets and Systems 51, 295-307 (1992)

11. Yager, R.R.: On ordered weighted averaging aggregation operators in multi-criteria decision making. Fuzzy Sets and Systems 2, 243-260 (1983)

12. Yager, R.R.: Quantifier guided Aggregating using OWA operators. International Journal of Intelligent Systems 1, 49-73 (1996)

13. Yager, R.R., Kreinovich, V.: On how to merge sorted lists coming from different web search tools. Soft Computing Research Journal 3(1), 83-88 (1999)

14. Yager, R.R., Rybalov, A.: Full reinforcement operators in aggregation techniques. IEEE Transactions on Systems, Man and Cybernetics 28, 757-769 (1998)

15. Zimmermann, H.-J., Zysno, P.: Latent connectives in human decision making. Fuzzy Sets and Systems 4, 37-51 (1980)

16. Bollmann, P., Raghavan, V.V., Jung, G.S., Shu, L.C.: On probabilistic notions of precision as a function of recall. In: Information Processing and Management, pp. 291-315. Pergamon Press, Tarrytown (1992) 